\title{
Influence of different mechanical stimuli in a multi-scale mechanobiological isotropic model for bone remodelling
}

\author{
E.G.F. Mercuri ${ }^{a, b}$, A.L. Daniel ${ }^{b}$, M.B. Hecke ${ }^{b}$, L. Carvalho ${ }^{c, *}$ \\ a Department of Environmental Engineering, Federal University of Paraná, Curitiba, Brazil \\ ${ }^{\mathrm{b}}$ Bioengineering Group, Post Graduate Program in Numerical Methods in Engineering, Federal University of Parana, Curitiba, Paraná, Brazil \\ ${ }^{c}$ INESC-TEC and Faculty of Sciences of the University of Porto (FCUP), Rua do Campo Alegre, 687, 4150-179 Porto, Portugal
}

\section{A R T I C L E I N F O}

\section{Article history:}

Received 9 November 2015

Revised 11 February 2016

Accepted 23 April 2016

\section{Keywords:}

Bone remodelling

Strain energy density

Multi-scale modelling

Apparent density

Femur

Mechanical stimuli

\begin{abstract}
A B S T R A C T
This work represents a study of a mathematical model that describes the biological response to different mechanical stimuli in a cellular dynamics model for bone remodelling. The biological system discussed herein consists of three specialised cellular types, responsive osteoblasts, active osteoblasts and osteoclasts, three types of signalling molecules, transforming growth factor beta (TGF- $\beta$ ), receptor activator of nuclear factor kappa-b ligand (RANKL) and osteoprotegerin (OPG) and the parathyroid hormone (PTH). Three proposals for mechanical stimuli were tested: strain energy density (SED), hydrostatic and deviatoric parts of SED. The model was tested in a two-dimensional geometry of a standard human femur. The spatial discretization was performed by the finite element method while the temporal evolution of the variables was calculated by the 4 th order Runge-Kutta method. The obtained results represent the temporal evolution of the apparent density distribution and the mean apparent density and thickness for the cortical bone after 600 days of remodelling simulation. The main contributions of this paper are the coupling of mechanical and biological models and the exploration of how the different mechanical stimuli affect the cellular activity in different types of physical activities. The results revealed that hydrostatic SED stimulus was able to form more cortical bone than deviatoric SED and total SED stimuli. The computational model confirms how different mechanical stimuli can impact in the balance of bone homeostasis.
\end{abstract}

(c) 2016 IPEM. Published by Elsevier Ltd. All rights reserved.

\section{Introduction}

Osteoporotic fractures are a major public health problem, with a high prevalence worldwide [1,2], especially in the hip joint and in particular are a serious problem in Brazil [3]. Hip fracture is the major consequence of osteoporosis among elderly people, in many Western nations, leading to chronic pain, disability, lower quality of life and lower mortality [1,4,5]. Many prospective studies have shown that bone mineral density (BMD) measurements are able to predict fracture [6].

Bone tissue is a dynamic system capable of changing its own density, in response to different biomechanical stimuli. The "mechanostat" theory of Frost states that bone adapts its strength to keep the strain, caused by physiological loads, in a certain interval [7]. Some authors consider that this interval lies outside of a "dead zone" and established that range was between 1000 and 2000 micro strain [8]. If strain is above this interval, new

* Corresponding author. Tel.: +351 220402 301; fax: +351220 402437.

E-mail address: licasc@gmail.com, lidia.r.carvalho@inesctec.pt (L. Carvalho). bone is formed, while below this interval, bone is resorbed. Osteocytes, are strain-sensitive cells and can transduce mechanical signals to groups of specialised cells, such as osteoblasts and osteoclasts, which are responsible for forming and resorbing bone matrix [9].

In the last decades, several research groups have worked in the development of new models, to describe the bone remodelling process, taking into account different stimuli in bone cell regulation, like mechanical strain, microdamage, cell biology, metabolic factors and other external contributions $[8,10]$. From a biochemical point of view, the first model correlated the differential activity of parathyroid hormone (PTH) as a regulator for bone resorption and formation. For example, Kroll et al. [11] found that an external administration of PTH can affect directly the time evolution of bone cells populations. Then, it was the time to demonstrate the role of hormones like autocrine and paracrine in the regulation of bone remodelling. Finally, a signalling pathway known as RANK/RANKL/OPG to regulate bone cells activities [10]. It was also found that this signalling pathway RANK-RANKL-OPG is an important regulation of the paracrine interactions between osteoblasts and osteoclast [12-15]. Wht is a secreted family of 
glycoproteins and its pathway signalling, particularly through low-density lipoprotein receptor-related protein 5 (LRP5), is very important in the regulation of bone mass and strength. Also, the Wnt $/ \beta$-catenin signalling is a normal physiological response to mechanical load, and that the activation of $\mathrm{Wnt} / \beta$-catenin pathway can enhance the sensitivity of osteoblasts/osteocytes to mechanical loading [16,17]. Osteoprogenitor cells, stromal cells, osteoblasts, and osteocytes are the candidates for sensing and responding to mechanical stimulus. Different approaches were considered to describe the mechanisms through which bone cells are able to sense their mechanical environment, like direct matrix deformations $[7,8]$, pressure and transient microdamage [8], accelerations, pressure waves, interstitial fluid flow, fluid drag forces, fluid shear stresses, or dynamic electric fields [18-20]. It is not clear, however, which of these stimuli are the most relevant for bone remodelling. Recently, authors developed numerical models that consider mechanoregulation functions that affect cellular activities based on the SED intensity [21]. The deviatoric and hydrostatic modes of SED interact with each other in a general anisotropic elastic material, as bone hard tissue is sometimes characterised [22]. However, in our study it was considered isotropic, linear elastic, material, so the hydrostatic and deviatoric modes of SED are non-interactive and the separation of the influence of both physical quantities is acceptable.

The aim of this study was to study the influence of different modes of the SED stimuli, in a cellular interaction model for the bone remodelling process. The main goal was to verify if the algorithm was able to predict the formation of the cortical mid diaphysis region, starting from a femur with homogeneous apparent density distribution. This is a common condition in bone remodelling simulations [23-25] and it was chosen to perform the validation of our model. The mechanical stimuli considered within the study, to update the physical properties of bone, were: SED, hydrostatic SED and deviatoric SED. Were also objectives of this study estimate the average thickness of cortical bone with different mechanical stimuli and calculate the mean apparent density of cortical and trabecular bone, after 1200 days of remodelling simulation, for each considered stimulus.

\section{Equations of the model}

The system of ordinary differential equations governing the coupling between osteoclasts and osteoblasts proposed by Lemaire et al. [13], has been improved by several authors [12-15,21,26].

The principal equations of the model are shown below, Eqs. (1-4) $[21,26]$. The variables $R, B, C$ represent the concentrations of pre-osteoblasts, active osteoblasts and active osteoclasts, respectively, and the quantity BV represents bone volume. The variables vary with respect to time $t$ and the first order ordinary differential equations of the system are displayed below.

$\frac{d R}{d t}=D_{R} \cdot \pi_{C}+P_{R} \cdot R \cdot \Pi_{w}-\frac{D_{B}}{\pi_{C}} \cdot R$

$\frac{d B}{d t}=\frac{D_{B}}{\pi_{C}} \cdot R-K_{B} \cdot B$

$\frac{d C}{d t}=D_{C} \cdot \pi_{L}-D_{A} \cdot \pi_{C} C$

$$
\frac{d \mathrm{VB}}{d t}=K_{\mathrm{form}} \cdot B-K_{\mathrm{res}} \cdot C
$$

In addition, the influence of mechanical stimulus, in the concentration of pre-osteoblast cells, is made by the following function:

$\Pi_{w}=\Pi_{w_{\text {equil }}}\left[1+\lambda\left(\frac{w}{w_{\text {equil }}}-1\right)\right]$

The terms related to RANKL are as follows:

$\pi_{L}=\left(\frac{K_{3}}{K_{4}}\right) \frac{K_{L}^{P} \pi_{P} B}{1+\frac{K_{3}}{K_{4}}+\frac{K 1}{K_{2} K_{0}}\left(\frac{K_{0}^{P}}{\pi_{P}+I_{0}}\right)}\left(1+\frac{I_{L}+P_{\mathrm{RANKL}_{\mathrm{w}}}}{r_{L}}\right)$

$P_{\text {RANKL }_{\mathrm{w}}}=K\left(1-\frac{w}{w_{\text {equil }}}\right)$

The term related to PTH is:

$\pi_{P}=\frac{\frac{I_{P}}{K_{P}}+\frac{S_{P}}{K_{P}}}{\frac{I_{P}}{K_{P}}+\frac{K_{6}}{K_{5}}}$

and the term representing the influence of TGF- $\beta$ is:

$\pi_{C}=\frac{C+f_{0} C^{S}}{C+C^{S}}$

All parameters of the model are provided in Table 1, as well as a brief explanation of the units used.

The primary difference incorporated into Scheiner's model [21] was the use of two limits for remodelling, an upper ( $\left.w_{\text {sup }}\right)$ and a lower $\left(w_{\text {inf }}\right)$ limit, with an intermediate "dead zone" indicating a region of equilibrium [27]. The numerical values of the constants $w_{\text {sup }}$ and $w_{\text {inf }}$ and all parameters are shown in Mercuri et al. [26].

\section{Strain energy density}

When an elastic solid is deformed by an applied force, the work produced by the surface and body forces is stored within the solid under the form of deformation energy. For an ideal elastic body, this energy is completely recovered after removing the load $[28,29]$. The strain energy can be written as:

$w=\frac{1}{2} \varepsilon_{i j} \sigma_{i j}$

$w=\frac{1}{2} \sigma \varepsilon$

$w=\frac{1}{2}\left(\begin{array}{lll}\varepsilon_{x x} & \varepsilon_{x y} & \varepsilon_{x z} \\ \varepsilon_{y x} & \varepsilon_{y y} & \varepsilon_{y z} \\ \varepsilon_{z x} & \varepsilon_{z y} & \varepsilon_{z z}\end{array}\right) \cdot\left(\begin{array}{lll}\sigma_{x x} & \sigma_{x y} & \sigma_{x z} \\ \sigma_{y x} & \sigma_{y y} & \sigma_{y z} \\ \sigma_{z x} & \sigma_{z y} & \sigma_{z z}\end{array}\right)$

$w=\frac{1}{2}\left(\varepsilon_{x x} \sigma_{x x}+2 \varepsilon_{x y} \sigma_{x y}+\varepsilon_{y y} \sigma_{y y}+2 \varepsilon_{y z} \sigma_{y z}+\varepsilon_{z z} \sigma_{z z}+2 \varepsilon_{x z} \sigma_{x z}\right)$

$w=\frac{1}{2}\left(\varepsilon_{x x} \sigma_{x x}+\varepsilon_{y y} \sigma_{y y}+\varepsilon_{z z} \sigma_{z z}\right)+\left(\varepsilon_{x y} \sigma_{x y}+\varepsilon_{y z} \sigma_{y z}+\varepsilon_{x z} \sigma_{x z}\right)$

The SED in the case of a plane stress state, $\sigma_{z z}=\sigma_{y z}=\sigma_{x z}=0$, is defined by Eq. (15) [28]:

$w=\frac{1}{2}\left(\varepsilon_{x x} \sigma_{x x}+\varepsilon_{y y} \sigma_{y y}+\varepsilon_{z z} \sigma_{z z}\right)+\varepsilon_{x y} \sigma_{x y}$

in which $\sigma_{x x}, \sigma_{y y}$ and $\sigma_{x y}$ are the stress tensor components and $\varepsilon_{x x}, \varepsilon_{y y}$ and $\varepsilon_{x y}$ are the deformation tensor components, in the Cartesian coordinate system.

Any tensor can be decomposed into deviatoric and hydrostatic parts, so the strain tensor can be written as:

$\varepsilon=\operatorname{hyd}(\varepsilon)+\operatorname{des}(\varepsilon)$ 
Table 1

Parameters of the simulation.

\begin{tabular}{|c|c|c|c|}
\hline Symbol & Unit & Value & Description \\
\hline$R_{0}$ & $\mathrm{Pm}$ & $0.0,007,734$ & Initial population of pre-osteoblasts. \\
\hline$B_{0}$ & $\mathrm{pM}$ & $0.0,007,282$ & Initial population of osteoblasts \\
\hline$C_{0}$ & $\mathrm{pM}$ & $0.0,009,127$ & Initial population of osteoclasts \\
\hline $\mathrm{BV}_{0}$ & $\%$ & $50 \%$ & Initial percentage of bone volume. \\
\hline$C^{S}$ & $\mathrm{pM}$ & 0.5 & Population of osteoclasts to obtain half differentiation flux \\
\hline$D_{A}$ & day $^{-1}$ & $2.660,768$ & Rate of osteoclast apoptosis caused by TGF- $\beta$ \\
\hline$d_{B}$ & day $^{-1}$ & 0.7 & Differentiation rate of responsive osteoblasts \\
\hline$D_{C}$ & pM day ${ }^{-1}$ & $2.1 \times 10^{-3}$ & Differentiation rate of osteoclast precursors \\
\hline$D_{R}$ & $\mathrm{pM}$ day $^{-1}$ & $0.0,070,744$ & Differentiation rate of osteoblast progenitors \\
\hline$f_{0}$ & - & 0.05 & Fixed proportion \\
\hline$K$ & $\mathrm{pM}$ & $1.0 \times 10^{1}$ & Fixed concentration of RANK \\
\hline$k_{1}$ & $\mathrm{pM}$ day $^{-1}$ & $1.0 \times 10^{-2}$ & Binding rate of OPG-RANKL \\
\hline$k_{2}$ & day $^{-1}$ & $1.0 \times 10^{1}$ & Unbinding rate of OPG-RANKL \\
\hline$k_{3}$ & $\mathrm{pM}$ day $^{-1}$ & $5.8 \times 10^{-4}$ & Binding rate of RANK-RANKL \\
\hline$k_{4}$ & day $^{-1}$ & $1.7 \times 10^{-2}$ & Unbinding rate of RANK-RANKL \\
\hline$k_{5}$ & pM day ${ }^{-1}$ & 0.02 & Binding rate of PTH with its receptor \\
\hline$k_{6}$ & day $^{-1}$ & 3.0 & Unbinding rate between PTH and its receptor \\
\hline$k_{B}$ & day $^{-1}$ & 0.7184 & Rate of active osteoblast elimination \\
\hline$K_{L}^{P}$ & pM cell ${ }^{-1}$ & $3.0 \times 10^{6}$ & Maximum quantity of RANKL on the surface of each cell \\
\hline$K_{0}$ & day $^{-1}$ & 0.35 & Rate of OPG elimination \\
\hline$K_{0}^{P}$ & pM day ${ }^{-1}$ cell $^{-1}$ & $2.0 \times 10^{5}$ & Minimum production rate of OPG per cell \\
\hline$k_{P}$ & day $^{-1}$ & 86.0 & Rate of PTH elimination \\
\hline$r_{L}$ & $\mathrm{pM}$ day $^{-1}$ & $1.0 \times 10^{3}$ & Rate of RANKL production and elimination \\
\hline$S_{P}$ & pM day ${ }^{-1}$ & 250.0 & Rate of systemic PTH synthesis \\
\hline$k_{\text {res }}$ & $\%$ cell $^{-1}$ & $0.20 \times 10^{2}$ & Relative rate of bone resorption per osteoclast \\
\hline$k_{\text {form }}$ & $\%$ cell $^{-1}$ & $0.3409 \times 10^{2}$ & Rate of bone deposition per osteoblast \\
\hline$P_{R}$ & $\mathrm{pM}_{\text {day }}^{-1}$ & 0.1694 & Proliferation rate of responsive osteoblasts \\
\hline$\lambda$ & - & 1.2 & Parameter to correct the proliferation rate of $R$ \\
\hline$\prod_{w_{\text {equil }}}$ & - & 1.2 & Equilibrium value for the mechanoregulatory function \\
\hline$\kappa$ & $\mathrm{pM}_{\text {day }}{ }^{-1}$ & $5.0 \times 10^{2}$ & Parameter to correct the production of RANKL by mechanical stimulus \\
\hline$W_{\text {inf }}$ & $\mathrm{Pa}$ & 20.0 & Lower limit of deformation energy \\
\hline$W_{\text {sup }}$ & $\mathrm{Pa}$ & 40.0 & Upper limit of deformation energy \\
\hline$w_{\text {equil. }}$ & $\mathrm{Pa}$ & 30.0 & Equilibrium deformation energy \\
\hline
\end{tabular}

The hydrostatic part of the strain tensor in Cartesian rectangular coordinates is:

$\operatorname{hyd}(\varepsilon)=\frac{1}{3} \operatorname{tr}(\varepsilon) I=\frac{1}{3} \varepsilon_{k k} \delta_{i j}=\frac{1}{3}\left(\varepsilon_{x x}+\varepsilon_{y y}+\varepsilon_{z z}\right) \delta_{i j}$

being $\boldsymbol{I}$ the identity tensor and $\operatorname{tr}(\varepsilon)$ the sum of the elements, in the principal diagonal of the strain tensor. The deviatoric part of the strain tensor, in Cartesian rectangular coordinates is:

$\operatorname{des}(\varepsilon)=\varepsilon_{i j}-\frac{1}{3} \operatorname{tr}(\varepsilon) I=\varepsilon_{i j}-\frac{1}{3} \varepsilon_{k k} \delta_{i j}=\varepsilon_{i j}-\frac{1}{3}\left(\varepsilon_{x x}+\varepsilon_{y y}+\varepsilon_{z z}\right) \delta_{i j}$

In terms of the principal directions, the expressions reduce to:

$\operatorname{hyd}(\varepsilon)=\frac{1}{3} \operatorname{tr}(\varepsilon) I=\frac{1}{3} \varepsilon_{k k} \delta_{i j}=\frac{1}{3}\left(\varepsilon_{1}+\varepsilon_{2}+\varepsilon_{3}\right) \delta_{i j}$

$\operatorname{des}(\varepsilon)$

$=\left(\begin{array}{ccc}\frac{2}{3} \varepsilon_{1}-\frac{1}{3}\left(\varepsilon_{2}+\varepsilon_{3}\right) & 0 & 0 \\ 0 & \frac{2}{3} \varepsilon_{2}-\frac{1}{3}\left(\varepsilon_{1}+\varepsilon_{3}\right) & 0 \\ 0 & 0 & \frac{2}{3} \varepsilon_{3}-\frac{1}{3}\left(\varepsilon_{1}+\varepsilon_{2}\right)\end{array}\right)$

The same decomposition and algebra performed to the strain tensor was applied to the stress tensor. The scalar quantity of interest, SED, can be decomposed into hydrostatic and deviatoric parts also. The hydrostatic part of the SED in terms of the Cartesian rectangular coordinates is:

$$
\begin{aligned}
\operatorname{hyd}(w) & =\frac{1}{2} \operatorname{hyd}(\varepsilon) \operatorname{hyd}(\sigma) \\
& =\frac{1}{2} \frac{1}{3}\left(\varepsilon_{x x}+\varepsilon_{y y}+\varepsilon_{z z}\right) \delta_{i j} \cdot \frac{1}{3}\left(\sigma_{x x}+\sigma_{y y}+\sigma_{z z}\right) \delta_{i j}
\end{aligned}
$$

Evaluating the dot product and simplifying the expression above:

$$
\begin{aligned}
\operatorname{hyd}(w)= & \frac{1}{6}\left(\varepsilon_{x x} \sigma_{x x}+\varepsilon_{x x} \sigma_{y y}+\varepsilon_{x x} \sigma_{z z}+\varepsilon_{y y} \sigma_{x x}+\varepsilon_{y y} \sigma_{y y}+\varepsilon_{y y} \sigma_{z z}\right. \\
& \left.+\varepsilon_{z z} \sigma_{x x}+\varepsilon_{z z} \sigma_{y y}+\varepsilon_{z z} \sigma_{z z}\right)
\end{aligned}
$$

For the case of a plane stress state, the hydrostatic part of the SED is:

$$
\operatorname{hyd}(w)=\frac{1}{6}\left(\varepsilon_{x x} \sigma_{x x}+\varepsilon_{x x} \sigma_{y y}+\varepsilon_{y y} \sigma_{x x}+\varepsilon_{y y} \sigma_{y y}+\varepsilon_{z z} \sigma_{x x}+\varepsilon_{z z} \sigma_{y y}\right)
$$

Furthermore, in terms of principal directions in a plane stress state where $\sigma_{3}=0$, we obtain:

$\operatorname{hyd}(w)=\frac{1}{6}\left(\varepsilon_{1} \sigma_{1}+\varepsilon_{1} \sigma_{2}+\varepsilon_{2} \sigma_{1}+\varepsilon_{2} \sigma_{2}+\varepsilon_{3} \sigma_{1}+\varepsilon_{3} \sigma_{2}\right)$

The deviatoric SED scalar quantity may be represented as:

$\operatorname{des}(w)=\frac{1}{2} \operatorname{des}(\varepsilon) \operatorname{des}(\sigma)$

The Eqs. (26) and (27) represent the deviatoric SED in rectangular coordinates and principal directions, respectively:

$$
\begin{aligned}
\operatorname{des}(w)= & \frac{1}{3}\left(\sigma_{x x} \varepsilon_{x x}+\sigma_{y y} \varepsilon_{y y}+\sigma_{z z} \varepsilon_{z z}\right) \\
& -\frac{1}{6}\left(\sigma_{x x} \varepsilon_{y y}+\sigma_{x x} \varepsilon_{z z}+\sigma_{y y} \varepsilon_{x x}+\sigma_{y y} \varepsilon_{z z}+\sigma_{z z} \varepsilon_{x x}+\sigma_{z z} \varepsilon_{y y}\right) \\
& +2\left(\sigma_{x y} \varepsilon_{x y}+\sigma_{x z} \varepsilon_{x z}+\sigma_{y z} \varepsilon_{y z}\right)
\end{aligned}
$$

$$
\begin{aligned}
\operatorname{des}(w)= & \frac{1}{3}\left(\sigma_{1} \varepsilon_{1}+\sigma_{2} \varepsilon_{2}+\sigma_{3} \varepsilon_{3}\right) \\
& -\frac{1}{6}\left(\sigma_{1} \varepsilon_{2}+\sigma_{1} \varepsilon_{3}+\sigma_{2} \varepsilon_{1}+\sigma_{2} \varepsilon_{3}+\sigma_{3} \varepsilon_{1}+\sigma_{3} \varepsilon_{2}\right)
\end{aligned}
$$




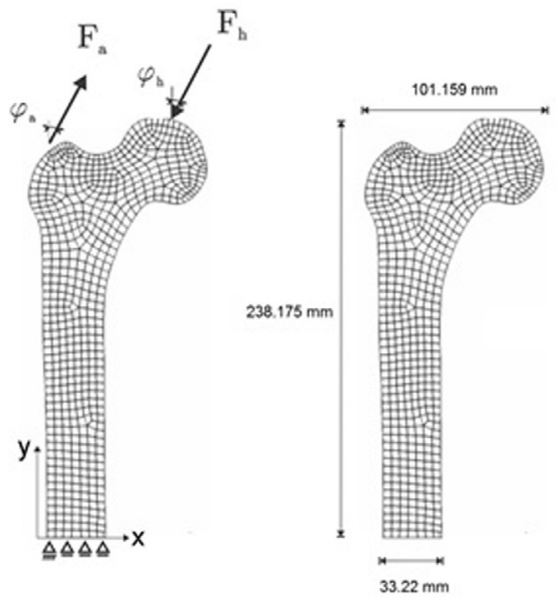

Fig. 1. Finite element mesh with its boundary conditions and dimensions.

The SED is frequently chosen as a biomechanical stimulus in computational studies of bone remodelling $[8,21,23,25]$.

\section{Materials and methods}

The algorithm used to simulate the cell populations was programmed inside the finite element code, in all nodes of the mesh. The size of the pre-osteoblast, osteoblast and osteoclast populations were saved in a vector for each node, and the previous population was used in the next day to calculate the new population, based on the new mechanical stimulus. The daily integration was performed by the RK4 method, for a total of 2500 days.

The mechanical influence on the cellular model is mediated according to the level of SED (w) in the bone tissue. The model considers that the osteoblast lineage can respond explicitly to SED stimuli. When the deformation energy is below the pre-defined limit $\left(w_{\text {inf }}\right)$, the $P_{\text {RANKL,w }}$ function causes a signalling of the cytosine RANKL by the osteoblasts. However, when $w>w_{\text {sup }}$, the proliferation rate of the osteoblast precursors increases [15].

The multi-scale mechanobiological isotropic model for bone remodelling, based on the 3 types of SED stimuli, consists of a twodimensional transient analysis of the variation of the constitutive bone properties. The model was applied to the proximal region of a femur that was obtained through a standardised solid model. The model of the femur represents a normal adult, with no specific sex, which was taken from composite sawbone models, provided by the Biomechanics European Laboratory (BEL) Repository [30]. It was chosen a section in the coronal plane, from the central region of the proximal femur, to generate the $2 \mathrm{D}$ model. The finite element mesh contains 598 quadrilateral elements and 1979 nodes. The dimensions of the model are provided in Fig. 1. The mesh convergence test by Zienkiewicz-Zhu method [31,32] was performed with $5 \%$ of error. The definition of the geometry, generation of the finite element mesh, selection of material properties and application of boundary conditions were performed in the preprocessing step. The finite element method (FEM) was used to solve the equilibrium equation (weak form of the principle of virtual power) and provided the nodal displacements, used to calculate the nodal stresses and strains. In post-processing, the code calculates the SED and solves Eqs. (1-4), updating the mechanical properties to start a new load cycle. Each load cycle represents a day of exercise for a normal person [23]. Finally, after updating the properties of the elements in all load cycles, the post-processing generates tables and images, illustrating the results. All steps of the calculation, namely the pre-processing, processing and post-processing, were performed in MATLAB® software [33].
Table 2

Percentage of cortical and trabecular bone formed, for each stimulus, at time of 600 days.

\begin{tabular}{llll}
\hline Bone type Stimulus & SED & Deviatoric SED & Hydrostatic SED \\
\hline Cortical bone & $12.9 \%$ & $19.9 \%$ & $21.9 \%$ \\
Trabecular bone & $87.1 \%$ & $80.1 \%$ & $78.1 \%$ \\
\hline
\end{tabular}

For each day's iteration, the BV is changed with the new density, characterising a new elastic modulus. Eq. (28) describes the empirical relationship between the bone density, given in $\mathrm{g} / \mathrm{cm}^{3}$, and the elasticity modulus, given in (MPa) [34].

$E(\rho)=3790 \rho^{3}$

The relationship between the density and BV is linear; when $\mathrm{BV}=0 \%$, we obtain $\rho=0.0 \mathrm{~g} / \mathrm{cm}^{3}$, and when $\mathrm{BV}=100 \%$, we obtain the density equal to $\rho=2.0 \mathrm{~g} / \mathrm{cm}^{3}$ [35].

To avoid excessive remodelling (unrealistic values of apparent density), maximum and minimum limits were established for the apparent density, which were $\rho_{\min }=0.2 \mathrm{~g} / \mathrm{cm}^{3}$ and $\rho_{\max }=2.0 \mathrm{~g} / \mathrm{cm}^{3}$.

For the transient analysis, an incremental explicit time step scheme was used to evaluate the evolution of biological variables and apparent density, starting from homogeneous distribution of apparent density. During one time step, corresponding to 1 day, a static analysis was performed, assuming bone tissue as an isotropic linear elastic material in the plane stress state. At the end of a daily cycle, the elastic modulus of each node was updated, based on the cellular interaction, which was mediated by the nodal mechanical stimulus. The osteoclasts were responsible for resorbing the bone tissue, and the osteoblasts were responsible to form new bone tissue. The boundary conditions were: zero displacement in the vertical direction $(y)$ in the bottom line of the mesh and zero horizontal displacement $(x)$ of the left node of the same line as it is shown in Fig. 1 [23]. Two loads were applied: a traction force on the head of the femur of $702 \mathrm{~N}$, with an angle of $\varphi_{a}=28^{\circ}$ from the vertical axis (clockwise) and a compression force of $2317 \mathrm{~N}$, on the medial portion with an angle of $\varphi_{h}=24^{\circ}$ from the vertical axis (clockwise). The methodology for the application of loads, like place of application and order of magnitude were in accordance to those adopted by Huiskes et al. [23], representing the load on the femoral head made by the abductor muscles, in a normal day walking activity.

In order to evaluate the influence of the biomechanical parameters in our multi-scale mechanobiological isotropic model, three different stimuli were considered: (i) the total SED, from Eq. (15); (ii) the deviatoric part of SED, from Eq. (26); (iii) The hydrostatic part of SED, from Eq. (23).

Statistical analyses were performed in order to enable the comparison of the results obtained for the three stimuli. All statistical measures (percentage of bone formation, averages and standard deviations) were performed for the 3 stimuli, after 600 days of simulation and are depicted in Tables $2-4$. It was defined a threshold of apparent density $\rho$, for cortical bone of $\rho \geq 1.5 \mathrm{~g} / \mathrm{cm}^{3}$, and for trabecular bone $\rho<1.5 \mathrm{~g} / \mathrm{cm}^{3}$. The mean value of cortical thickness was calculated considering the average thickness of 5 measurements, in different points, made in the diaphysis. The points were equally distributed in the $y$ direction, spaced $36.5 \mathrm{~mm}$ between them.

\section{Results and discussion}

In Figs. 2 and 3 are displayed the distribution of the SED and apparent density respectively for days 1, 200, 400, 600, 800, 1000 and 1200 , for the three different stimuli considered in this study. The sampling of days, depicted in those figures, was considered 


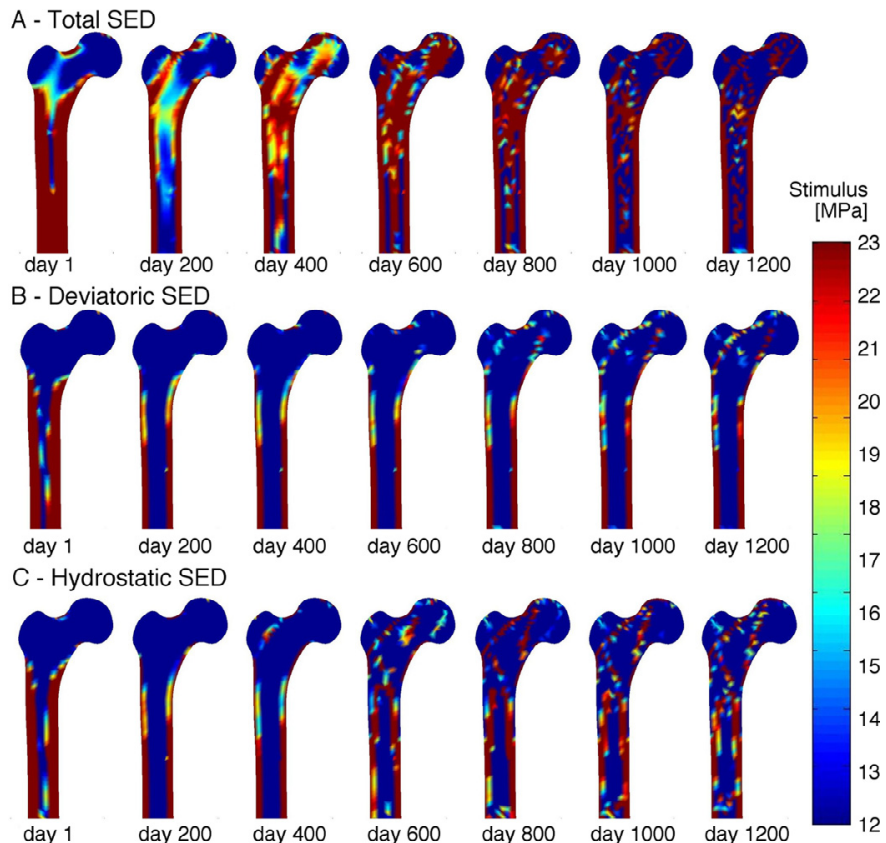

Fig. 2. Distribution of strain energy density for days $1,200,400,600,800,1000$ and 1200 for three stimuli types: (A) Total SED, (B) Deviatoric part of SED and (C) Hydrostatic part of SED.

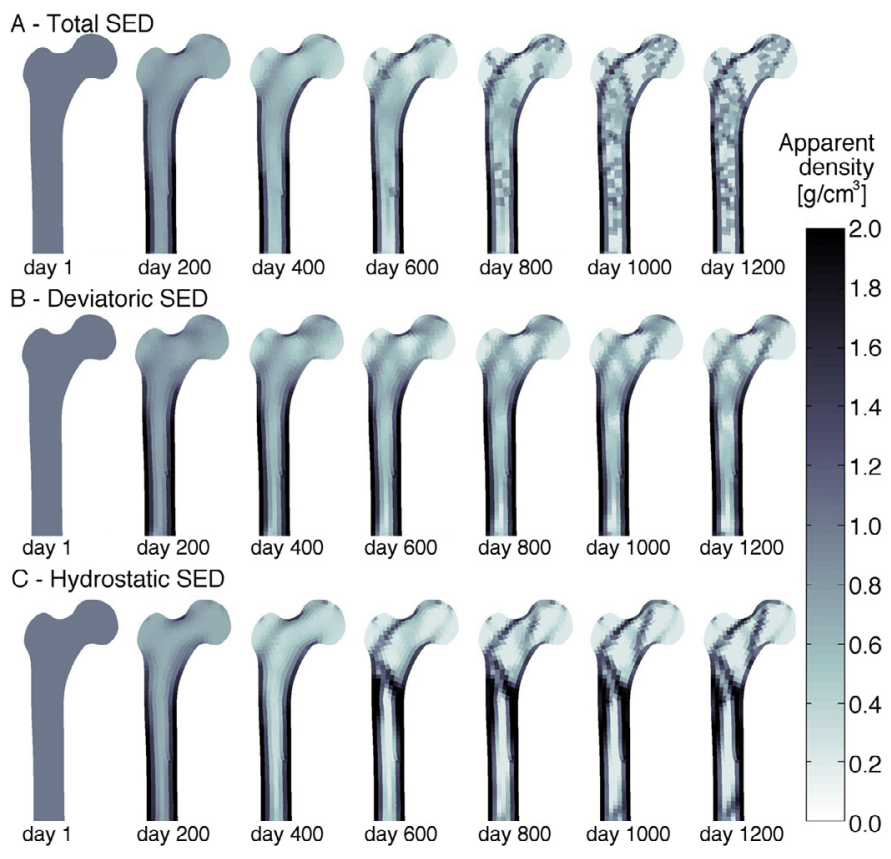

Fig. 3. Distribution of apparent density for days 1, 200, 400, 600, 800, 1000 and 1200 for three stimuli types: (A) Total SED, (B) Deviatoric part of SED and (C) Hydrostatic part of SED.

significant to reveal the evolution of the SED and the apparent density distribution in the bone tissue.

The deviatoric SED stimulus distribution after day 200 is concentrated mainly in the diaphysis and after 800 days it is also viewed in some regions of the epiphysis. The deviatoric SED stimulus is visually highly correlated with the formation of the cortical bone in the diaphysis. Moreover, the hydrostatic SED stimulus can be associated with the formation of the cortical bone in the metaphysis. After 600 days it has a more random distribution and can be related with the direction of the epiphyseal lines in the epi-
Table 3

Mid diaphysis cortical thickness average for 600 days.

\begin{tabular}{lll}
\hline Stimulus $\backslash$ Region & Left cortex $(\mathrm{mm})$ & Right cortex $(\mathrm{mm})$ \\
\hline SED & 3.80 & 4.75 \\
Deviatoric SED & 4.75 & 6.50 \\
Hydrostatic SED & 6.57 & 9.27 \\
\hline
\end{tabular}

Table 4

Apparent density average and standard deviation $\left(\mathrm{g} / \mathrm{cm}^{3}\right)$ for 600 days.

\begin{tabular}{llll}
\hline Bone type Stimulus & SED & Deviatoric SED & Hydrostatic SED \\
\hline Cortical Bone & $1.93 \pm 1.86$ & $1.88 \pm 1.84$ & $1.97 \pm 1.88$ \\
Trabecular Bone & $0.62 \pm 1.77$ & $0.68 \pm 1.77$ & $0.60 \pm 1.69$ \\
\hline
\end{tabular}

physis. The total SED is uniformly distributed along the diaphysis and after 400 days of remodelling simulation it is present in both metaphysis and epiphysis. In the first day of simulation, the total and deviatoric SED stimuli are present locally, near the application of the compression force, and for all stimuli it spreads along the femur head with the progression of the time step scheme.

The obtained results for the apparent density distribution, after 200 days, are in accordance with other numerical results in literature $[24,27,36]$ and can be also qualitatively compared with the image of an X-ray of a natural proximal femur [27]. After 800 days, the apparent density distribution, considering the total SED stimulus, exhibits the phenomenon known as checker boarding, also found in the literature [36]. However, this numeric instability does not appear in the apparent density distribution for the other two stimuli, the deviatoric SED and hydrostatic SED. The apparent density distribution for the deviatoric SED stimulus has the most uniform distribution.

In Table 2 are displayed the results corresponding to the percentage of cortical and trabecular bones formed, for the three stimuli considered. It can be seen that it is higher for the hydrostatic SED stimulus, followed by deviatoric SED stimulus and finally by SED stimuli. The ratio of trabecular and cortical bone formation is 6.8 for SED stimulus, 4.0 for deviatoric SED stimulus and 3.6 for hydrostatic SED stimulus.

The average thickness of the right cortex was higher than the left cortex, for all stimuli, after 600 days of bone remodelling simulation, as it is shown in Table 3. This can be explained by the fact that the applied compression force, in the right part of the femur head, has one order of magnitude higher than the traction force, applied in the left part of the femur head.

The cortical bone formed considering the hydrostatic SED stimulus is more dense or compact than that formed with the SED and deviatoric SED stimuli. Table 4 resumes the average and standard deviation of the apparent density, for both cortical and trabecular bone, after 600 days of bone remodelling simulation. After 600 days the remodelling patterns begin to show some unrealistic distributions of apparent density.

All the obtained results indicate that the developed multi-scale mechanobiological isotropic model for bone remodelling, implemented with the finite element, for the three different stimuli considered, can achieve reliable results for bone remodelling in the first 600 days of simulation and are in accordance with other results in literature $[24,27,36]$. The cortex average thickness and average apparent density of cortical and trabecular bone lie within the limits described in experimental measurements [37-44].

\section{Conclusions}

The comparison of the three different mechanical stimuli in the multi-scale mechanobiological isotropic model revealed different evolutionary patterns for bone remodelling. 
Classical biomechanical models rarely use systems of differential equations to describe the local behaviour of biological variables; often authors use phenomenological laws that disguise how the remodelling process occurs. The major contribution of this study is the coupling of different types of mechanical stimuli with the biological response for bone remodelling. The results show that the local activity of the cells can transform the stimuli into a natural distribution of the apparent density.

The research represents a hypothetical simulation of formation of the cortex in the cortical mid diaphysis region that mimics the natural morphology of bone. Hydrostatic SED stimulus formed thicker mid diaphysis cortical thickness than deviatoric SED stimulus. This was caused by some factors like the geometrical characteristic of the femur (long bone), the path of dissipation of the strains and stresses in the model and finally the intensity and direction of the force boundary condition distributed over the femur head.

This study gives some insight concerning the bone turnover phenomenon and how computational tools can examine bone pathologies, bone healing and new drugs. Further studies need to be performed with a corroboration of experimental data to develop more complete models with the addition of new biological variables to represent hormonal variations and other proteins related to bone biology and related diseases.

\section{Future work}

There are large number of bone diseases that are related to the type of mechanical stress applied to the tissue. The levels of mechanical stimulus sensed by cells and at tissue scale are different due to the hierarchical characteristics of bone, thus macro and microscale measurements of stresses and strains are necessary to understand how cells respond to stimuli. One limitation of our physical model is the lack of information of pore pressure and fluid flow in the lacunae and canaliculi structures, which have a direct impact in the sensors of the cytoplasmic membrane of the cells. In a next step, it is expected to include this information, to improve our model, trying to achieve better results. Another suggestion for future works should include investigation of other loads intensities or load cases and a three dimensional geometry.

In the future, these developed computational tools can be used to examine bone pathologies, bone healing and new drugs. Further studies need to be performed with a corroboration of experimental data to develop more complete models with the addition of new biological variables to represent hormonal variations and other proteins related to bone biology and related diseases.

\section{Conflict of interest}

The authors declare no conflict of interest.

\section{Competing interests}

None declared

\section{Funding}

National Council for Scientific and Technological Development (Conselho Nacional de Desenvolvimento Científico e Tecnológico, CNPq, Brazil) for funding (304058/2012-2 and 307927/2015-6).

\section{Ethical approval}

Not required

\section{Acknowledgements}

The authors acknowledge the Coordination for the Improvement of the Higher Level Education Personnel (Coordenação de Aperfeiçoamento de Pessoal de Nível Superior, CAPES) and the National Council for Scientific and Technological Development (Conselho Nacional de Desenvolvimento Científico e Tecnológico, CNPq) for the scholarships and funding.

\section{References}

[1] Dhanwal DK, Dennison EM, Harvey NC, Cooper C. Epidemiology of hip fracture: Worldwide geographic variation. Indian J Orthop 2011;45(1):15-22.

[2] Johnell O, Kanis JA. An estimate of the worldwide prevalence and disability associated with osteoporotic fractures. Osteoporos Int 2006;17:1726-33.

[3] Pinheiro MM, Ciconelli RM, Martini LA, Ferraz MB. Clinical risk factors for osteoporotic fractures in Brazilian women and men: the Brazilian Osteoporosis Study (BRAZOS). Osteoporos Int 2009;20(3):399-408.

[4] Castro da Rocha FA, Ribeiro AR. Low incidence of hip fractures in an equatorial area. Osteoporos Int 2003;14(6):496-9.

[5] Cooper C, Campion G, Melton LJ. Hip fractures in the elderly: a world-wide projection. Osteoporos Int 1992;2(6):285-9.

[6] Pinheiro MM, Castro CM, Szejnfeld VL. Low femoral bone mineral density and quantitative ultrasound are risk factors for new osteoporotic fracture and total and cardiovascular mortality: a 5-year population-based study of Brazilian elderly women. J Gerontol A Biol Sci Med Sci. 2006;61(2):196-203.

[7] Frost HM. Tetracycline-based histological analysis of bone remodelling. Calcif Tissue Res 1969;3(3):211-37.

[8] Mcnamara LM, Prendergast PJ. Bone remodeling algorithms incorporating both strain and microdamage stimuli. J Biomech 2007:40(6):1381-91.

[9] Robling AG, Castillo AB, Turner CH. Biomechanical and molecular regulation of bone remodeling. Ann Rev Biomed Eng 2006;8:455-98.

[10] Bougherara H, Klika V, Maršík F, Mařík IA, Yahia L. New predictive model for monitoring bone remodeling. J Biomed Mat Res A 2010;95(1):9-24.

[11] Kroll MH. Parathyroid hormone temporal effects on bone formation and resorption. Bull Math Biol 2000;62(1):163-88.

[12] Komarova SV, Smith RJ, Dixon SJ, Sims SM, Wahl LM. Mathematical model predicts a critical role for osteoclast autocrine regulation in the control of bone remodeling. Bone 2003;33(2):206-15.

[13] Lemaire V, Tobina FL, Grellera LD, Choa CR, Suva LJ. Modeling the interactions between osteoblast and osteoclast activities in bone remodeling. J Theoret Biol 2004;229(3):293-309.

[14] Pivonka P, Zimak J, Smith DW, Gardiner BS, Dunstan CR, Sims NA, et al. Model structure and control of bone remodeling: a theoretical study. Bone 2008;43(2):249-63.

[15] Pivonka P, Zimak J, Smith DW, Gardiner BS, Dunstan CR, Sims NA, et al. Theoretical investigation of the role of the RANK-RANKL-OPG system in bone remodeling. J Theor Biol 2010;262(2):306-16.

[16] Sawakami K, Robling AG, Ai M, Pitner ND, Liu D, Warden SJ, et al. The Wnt co-receptor LRP5 is essential for skeletal mechanotransduction but not for the anabolic bone response to parathyroid hormone treatment. J Biol Chem 2006;281(33):23698-711

[17] Robinson JA, Chatterjee-Kishore M, Yaworsky PJ, Cullen DM, Zhao W, Li C, et al. Wnt/beta-catenin signaling is a normal physiological response to mechanical loading in bone. J Biol Chem 2006;281(42):31720-8.

[18] Berk BC, Corson MA, Peterson TE, Tseng H. Protein kinases as mediators of fluid shear stress stimulated signal transduction in endothelial cells: a hypothesis for calcium-dependent and calcium- independent events activated by flow. Biomech 1995;28(12):1439-50.

[19] Malone AM, Batra NN, Shivaram G, Kwon RY, You L, Kim CH, et al. The role of actin cytoskeleton in oscillatory fluid flow-induced signaling in MC3T3-E1 osteoblasts. Am J Physiol Cell Physiol 2007;292(5):C1830-6.

[20] You L, Temiyasathit S, Lee P, Kim CH, Tummala P, Yao W, et al. Osteocytes as mechanosensors in the inhibition of bone resorption due to mechanical loading. Bone 2008;42(1):172-9.

[21] Scheiner S., Pivonka P., Hellmich C., Smith D. Mechanobiological regulation of bone remodeling-theoretical development of coupled systems biologymicromechanical approach. 2012arXiv1201.2488S. 2012.

[22] Cowin SC. Deviatoric and hydrostatic mode interaction in hard and soft tissue. J Biomech 1990;23(1):11-14.

[23] Huiskes R, Weinan H, Grootenboer HJ, Dalstra M, Fudala B, Slooff TJ. Adaptive bone-remodeling theory applied to prosthetic-design analysis. J Biomech 1987;20(11-12):1135-50.

[24] Jacobs CR. Numerical simulation of bone adaptation to mechanical loading (PhD Thesis). University of Stanford; 1994.

[25] Jacobs CR, Levenston ME, Beaupré GS, Simo JC, Carter DR. Numerical instabilities in bone remodeling simulations: the advantages of a node-based finite element approach. J Biomech 1995;28(4):449-59.

[26] Mercuri EGF, Daniel AL, Machado RD, Hecke MB. Application of a multi-scale mechanobiological model for bone remodeling. J Med Imag Health Inf 2014;4(1):142-6.

[27] Doblaré M, García JM. Anisotropic bone remodeling model based on a continuum damage-repair theory. J Biomech 2002;35(1):1-17. 
[28] Sadd MH. Elasticity: theory, applications, and numerics. 2nd ed. Elsevier; 2009. p. $103-18$.

[29] Reddy JN. Principles of continuum mechanics: a study of conservation principles with applications. 1st ed. Cambridge University Press; 2010. p. 196.

[30] Viceconti M, Casali M, Massari B, Cristofolini L, Bassini S, Toni A. The "standardized femur program" proposal for a reference geometry to be used for the creation of finite element models of the femur. J Biomech 1996;29(9):1241.

[31] Cook RD. Finite element modeling for stress analysis. 1st ed. John Wiley \& Sons, Inc; 1995.

[32] Zienkiewicz OC, Zhu JZ. A simple error estimator and adaptive procedure for practical engineering analysis. Int J Numer Methods Eng 1987;24:337-57.

[33] MATLAB Version 7.10.0 (2010b), The MathWorks, Inc., Natick, Massachusetts, United States, 2010.

[34] Carter DR, Hayes WC. The compressive behavior of bone as a two-phase porous structure. J Bone Joint Surg Am 1997;59(7):954-62.

[35] Taylor WR, Roland E, Ploeg H, Hertig D, Klabunde R, Warner MD, et al. Determination of orthotropic bone elastic constants using FEA and modal. J Biomech 2002;35(6):767-73.

[36] Hazelwood SJ, Martin RB, Rashid MM, Rodrigo JJ. A mechanistic model for internal bone remodeling exhibits different dynamic responses in disuse and overload. J Biomech 2001;34(3):299-308.
[37] Bousson V, Bergot C, Meunier A, Barbot F, Parlier-Cuau C, Laval-Jeantet AM, et al. CT of the mid diaphyseal femur: cortical bone mineral density and relation to porosity. Radiology 2000;217(1):179-87.

[38] Fyhrie DP, Carter DR. Femoral head apparent density distribution predicted from bone stresses. J Biomech 1990;23(1):1-10.

[39] Hangartner TN, Gilsanz V. Evaluation of cortical bone by computed tomography. J Bone Miner Res 1996;11(10):1518-25.

[40] Rodríguez-Soto AE, Fritscher KD, Schuler B, Issever AS, Roth T, Kamelger F, et al. Texture analysis, bone mineral density, and cortical thickness of the proximal femur: fracture risk prediction. J Comp Assist Tomogr 2010;34(6):949-57.

[41] Treece GM, Gee AH, Mayhew PM, Poole KES. High resolution cortical thickness measurement from clinical CT data. Med Image Anal 2010;14(3):276-90.

[42] Treece GM, Poole KES, Gee AH. Imaging the femoral cortex: thickness, density and mass from clinical CT. Med Image Anal 2012;16(5-4):952-65.

[43] Treece GM, Gee AH. Independent measurement of femoral cortical thickness and cortical bone density using clinical CT. Med Image Anal 2015;20(1):249-64.

[44] Yang L, Udall WJM, McCloskey EV, Eastell R. Distribution of bone density and cortical thickness in the proximal femur and their association with hip fracture in postmenopausal women: a quantitative computed tomography study. Osteoporos Int 2014;25(1):251-63. 